\title{
Avaliação da determinação do tempo de protrombina em amostras de sangue colhidas por duas diferentes técnicas
}

Primeira submissão em 25/06/04 Última submissão em 22/07/05 Aceito para publicação em 04/08/05 Publicado em 20/08/05

\section{Evaluation of determination of prothrombin time in blood samples using two different collection techniques}

Paulo Roberto de Melo Reis'; Mauro Meira de Mesquita'; Karlla Greick Batista Dias-Penna'; Frank de Souza Castro ${ }^{3}$; Fernando Amorim Balestra ${ }^{3}$

\begin{tabular}{l|l}
\multicolumn{1}{c|}{ Unitermos } & resumo \\
Protrombina & $\begin{array}{l}\text { O presente trabalho tem como objetivo avaliar a interferência de diferentes técnicas de coleta sanguínea } \\
\text { na determinação do tempo de protrombina. Duas técnicas de coleta foram utilizadas: uma, realiza- } \\
\text { Anticoagulante oral }\end{array}$ \\
$\begin{array}{l}\text { Vitamina K } \\
\text { dangramento }\end{array}$ & $\begin{array}{l}\text { valores obtidos na determinação do tempo de protrombina provenientes das duas técnicas de coleta } \\
\text { foram comparados. Foram analisadas amostras de pacientes que apresentavam tempo de protrombina } \\
\text { normais e anormais por estarem em tratamento oral com medicação anticoagulante. A conclusão foi } \\
\text { que o processo de coleta utilizando tanto o sistema a vácuo como a aspiração manual com seringa de } \\
\text { plástico não interfere no resultado do teste, e os valores obtidos, comparando-se os dois métodos de } \\
\text { coleta, não apresentaram diferenças estatisticamente significativas. }\end{array}$
\end{tabular}

abstract

The objective of the present study is to evaluate the interference of different blood collection techniques in determining prothrombin time. Two collection techniques were used. One of them uses the vacuum system and the other, manual aspiration using plastic syringe. The obtained values of determination of prothrombin time from these two techniques were compared. We analyzed patients' samples that presented normal and non-normal prothrombin time owing to oral treatment with anticoagulant medication. The conclusion was that the collection processes using either vacuum system or manual aspiration with plastic syringe to obtain samples destined to the prothrombin time analysis do not interfere in the test result and the obtained values. In comparing both collecting techniques we did not find any significant statistical differences. key words

Prothrombin

Oral anticoagulant

Vitamin $K$

Bleeding

Thromboembolism

\section{Introdução}

A hemostasia é o resultado do equilíbrio entre as proteínas pró-coagulantes e as anticoagulantes ${ }^{(13)}$. $\mathrm{O}$ rompimento desse equilíbrio pode acontecer com uso de anticoagulantes orais ou drogas antivitamina $\mathrm{K}$, que freqüentemente são administrados com o intuito de prevenir fenômenos tromboembólicos $(8,10,16)$.
Para que o tratamento com as drogas anticoagulantes orais não tenha efeitos adversos e para evitar a superdosagem, a dose do medicamento deve ser ajustada ao paciente e monitorada pela determinação do tempo de protrombina e da relação normatizada internacional $(\mathrm{RNI})^{(8,16)}$. O tempo de protrombina (TP) foi descrito por Quick, em 1935, para quantificar a

1. Biomédico; professor assistente da disciplina Hematologia Laboratorial do Departamento de Biomedicina da Universidade Católica de Coiás (UCC)

2. Biomédico; coordenador do Laboratório de Análises Clínicas do Departamento de Biomedicina da UCC.

3. Biomédico; pesquisador associado ao Departamento de Biomedicina da UCC. 
protrombina e avaliar os fatores II, V, VII e X da coagulação

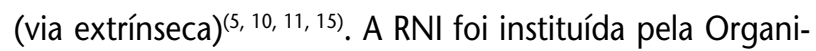
zação Mundial de Saúde (OMS), em 1983, e expressa a uniformização dos resultados, pois leva em consideração a sensibilidade do reagente (tromboplastina) utilizado no ensaio ${ }^{(2)}$, diminuindo, assim, as discrepâncias entre os resultados do tempo de protrombina, dando mais confiabilidade ao teste ${ }^{(2,8,16)}$. O cálculo da RNI só é necessário para pacientes com tempo de protrombina alterada pelo uso de anticoagulantes orais ${ }^{(11,16)}$.

O paciente em terapia anticoagulante oral necessita de monitoração rigorosa, envolvendo tanto fatores inerentes a ele quanto fatores relacionados à determinação do teste, tais como a coleta, a separação, o acondicionamento da amostra, o reagente e a realização do ensaio ${ }^{(2)}$. Cabe ao laboratório destinar cuidados especiais em todas as etapas do teste (desde a coleta, o processamento e o armazenamento das amostras, que fazem parte da fase pré-analítica; à realização do ensaio, que é a fase analítica; até a liberação do resultado, que faz parte da fase pós-analítica). Esses cuidados têm como objetivo a tentativa de excluir os fatores interferentes, mantendo a exatidão e a precisão inerentes ao analito ${ }^{(1-3,5,8,9,17)}$.

Em relação a TP e RNI, alguns estudos revelam que tanto os reagentes utilizados quanto $\mathrm{o}$ anticoagulante podem interferir nos resultados ${ }^{(12,14,19)}$. Quanto ao método de obtenção da amostra, parece não haver diferenças significativas entre a amostra capilar e a plasmática, embora os resultados não sejam uniformes entre os estudos ${ }^{(3,4,18)}$. No entanto, pouco se sabe a respeito da interferência dos recipientes de coleta imediatos em relação ao resultado do teste. $O$ objetivo deste trabalho é avaliar a interferência na determinação do tempo de protrombina em amostras de sangue colhidas por duas técnicas distintas.

\section{Material e métodos}

Foram analisadas 180 amostras de sangue obtidas de 90 pacientes. Determinou-se o tempo de protrombina em aparelho automatizado, coagulômetro compacto com memória para curva de calibração e resultados - utilizandose tromboplastina comercial (Wiener Lab. Lote: 306185) com índice de sensibilidade internacional (ISI) igual a 1,4, em pacientes clinicamente normais e em terapia anticoagulante oral. $\mathrm{O}$ estudo foi realizado no período compreendido entre agosto e dezembro de 2003, no Laboratório da Área de Saúde do Departamento de Biomedicina da Universidade Católica de Goiás. A participação no trabalho foi voluntária, mediante consentimento do paciente, que foi informado a respeito do estudo e assegurado o acesso ao resultado.

\section{Coleta com seringa e tubo de plástico}

A amostra foi obtida mediante punção venosa, atraumática, para evitar contaminação com líquidos tissulares, com seringa de plástico e agulha descartável 21G1 (25 x 8) e anticoagulante citrato de sódio $(18,8 \mathrm{~g} / \mathrm{dl})$ da Labtest Diagnóstica (lote: 2047), na proporção de uma gota do anticoagulante para $3 \mathrm{ml}$ de sangue, conforme orientação do fabricante. Posteriormente, a amostra foi colocada em recipiente de plástico, homogeneizada e centrifugada por dez minutos, a 3.000rpm, para a obtenção de plasma pobre em plaquetas.

\section{Coleta com sistema a vácuo}

A amostra foi obtida por meio de punção venosa, atraumática, porém em outro vaso sangüíneo, diretamente em tubo siliconizado do sistema Vacutainer (Becton-Dickinson), contendo $0,5 \mathrm{ml}$ de solução estéril de citrato de sódio (19,2mg de citrato de sódio e $2 \mathrm{mg}$ de ácido cítrico) e agulha descartável $21 \mathrm{G} 1(25 \times 8)$. O volume de sangue coletado foi de, aproximadamente, $4,5 \mathrm{ml}$, homogeneizado e centrifugado por dez minutos, a 3.000rpm, para obtenção de plasma pobre em plaquetas.

O plasma controle foi obtido entre os pacientes do próprio grupo em estudo. Para isso foram escolhidos, aleatoriamente, três pacientes saudáveis e que não estavam em uso de nenhuma forma de medicamento. A média do pool de amostras utilizada como referência para ambos os processos de obtenção da amostra foi de 12 segundos. Para comparação entre os dois métodos foi empregado o teste $t$ de Student pareado.

\section{Resultados}

A Tabela 1 compara os resultados do TP obtidos dos 90 pacientes estudados tanto pela técnica a vácuo quanto pela técnica da seringa. Dos 90 pacientes, 73 apresentavam-se hígidos, sem queixas de qualquer sintoma, sem história de outro resultado anterior alterado e não estavam em uso de medicação, portanto apresentaram o tempo de protrombina dentro dos limites da normalidade, e a diferença em cada determinação não apresentou discrepância, sendo que a maior diferença entre uma técnica de coleta e outra foi de 0,2 . 
\begin{tabular}{l|l} 
Tabela 1 & $\begin{array}{l}\text { Comparação dos resultados de TP em } 90 \text { pacientes estabelecidos após as duas técnicas de } \\
\text { coleta e comparação dos resultados de RNI nos } 17 \text { pacientes em uso de anticoagulante oral }\end{array}$ \\
\hline
\end{tabular}

\begin{tabular}{|c|c|c|c|c|c|c|c|c|c|c|}
\hline Paciente & $\begin{array}{c}\text { TP } \\
\text { vácuo }\end{array}$ & $\begin{array}{c}\mathrm{TP} \\
\text { seringa }\end{array}$ & Paciente & $\begin{array}{c}\text { TP } \\
\text { vácuo }\end{array}$ & $\begin{array}{c}\mathrm{TP} \\
\text { seringa }\end{array}$ & Paciente & $\begin{array}{c}\text { TP } \\
\text { vácuo }\end{array}$ & $\begin{array}{l}\text { TP se- } \\
\text { ringa }\end{array}$ & $\begin{array}{l}\text { RNI } \\
\text { vácuo }\end{array}$ & $\begin{array}{c}\text { RNI } \\
\text { seringa }\end{array}$ \\
\hline 1 & 14,5 & 13,8 & 31 & 14,6 & 14,6 & 61 & 13,8 & 13,8 & - & - \\
\hline 2 & 13,3 & 13 & 32 & 12,8 & 12,8 & 62 & 13,6 & 13,6 & - & - \\
\hline 3 & 12,6 & 12,8 & 33 & 12,5 & 12,5 & 63 & 14,5 & 14,5 & - & - \\
\hline 4 & 14,5 & 12,9 & 34 & 15 & 15 & 64 & 14,2 & 14,2 & - & - \\
\hline 5 & 14,1 & 13,6 & 35 & 12,8 & 12,8 & 65 & 13,8 & 13,8 & - & - \\
\hline 6 & 12,8 & 12,8 & 36 & 12,6 & 12,6 & 66 & 13,9 & 13,9 & - & - \\
\hline 7 & 12,9 & 12,7 & 37 & 14,6 & 14,6 & 67 & 12,7 & 12,7 & - & - \\
\hline 8 & 12,6 & 13,2 & 38 & 14,8 & 14,8 & 68 & 13,4 & 13,4 & - & - \\
\hline 9 & 13,5 & 13,7 & 39 & 13 & 13 & 69 & 12,2 & 12,2 & - & - \\
\hline 10 & 14,2 & 13,9 & 40 & 14 & 14 & 70 & 13,7 & 13,7 & - & - \\
\hline 11 & 13,6 & 14 & 41 & 14,2 & 14,2 & 71 & 12,2 & 12,2 & - & - \\
\hline 12 & 13,8 & 12,8 & 42 & 13 & 13 & 72 & 11,7 & 11,7 & - & - \\
\hline 13 & 12,9 & 13,1 & 43 & 13,4 & 13,4 & 73 & 12,9 & 12,9 & & \\
\hline 14 & 14 & 13,2 & 44 & 13,2 & 13,2 & 74 & 35,2 & 36,2 & 4,7 & 4,5 \\
\hline 15 & 12 & 13 & 45 & 13,3 & 13,3 & 75 & 16,6 & 17,2 & 1,7 & 1,6 \\
\hline 16 & 14 & 13,8 & 46 & 12,5 & 12,5 & 76 & 20 & 18,8 & 1,9 & 2 \\
\hline 17 & 15,7 & 15,8 & 47 & 14,4 & 14,4 & 77 & 17,4 & 19,4 & 2 & 1,7 \\
\hline 18 & 12,8 & 13,3 & 48 & 13,6 & 13,6 & 78 & 25,9 & 25,2 & 2,8 & 2,9 \\
\hline 19 & 13 & 14 & 49 & 12,8 & 13,6 & 79 & 17,1 & 17,8 & 1,7 & 1,6 \\
\hline 20 & 12,6 & 12,6 & 50 & 12,4 & 12,4 & 80 & 33,9 & 30,4 & 3,7 & 4,3 \\
\hline 21 & 13,3 & 13,2 & 51 & 12,4 & 12,4 & 81 & 26,2 & 27,2 & 3,1 & 3 \\
\hline 22 & 13,2 & 13,2 & 52 & 12,5 & 12,5 & 82 & 16,4 & 17,4 & 1,7 & 1,5 \\
\hline 23 & 12,6 & 12,8 & 53 & 14,1 & 14,1 & 83 & 24,7 & 26,6 & 3 & 2,7 \\
\hline 24 & 12,9 & 12,9 & 54 & 14,7 & 14,7 & 84 & 28,8 & 27,2 & 3,1 & 3,4 \\
\hline 25 & 12,8 & 13,6 & 55 & 12,6 & 12,6 & 85 & 34,3 & 33 & 4,1 & 4,4 \\
\hline 26 & 15,6 & 15,6 & 56 & 13,6 & 13,6 & 86 & 23 & 23 & 2,5 & 2,5 \\
\hline 27 & 12,6 & 12,6 & 57 & 12,5 & 12,5 & 87 & 31,2 & 31,2 & 3,8 & 3,8 \\
\hline 28 & 13,6 & 13,6 & 58 & 13,4 & 13,4 & 88 & 21,4 & 20,4 & 2,1 & 2,2 \\
\hline 29 & 12,6 & 12,6 & 59 & 12,4 & 12,4 & 89 & 18,9 & 18,8 & 1,9 & 1,9 \\
\hline 30 & 13,2 & 13,2 & 60 & 12,6 & 12,6 & 90 & 27,1 & 27,5 & 3,2 & 3,1 \\
\hline
\end{tabular}

Os dados em negrito são dos pacientes em uso de anticoagulante oral.

Além da comparação dos valores do TP, a Tabela 1 compara também os valores da RNI para os outros 17 pacientes que estavam em uso de anticoagulante oral. Para esses pacientes foram feitos os cálculos da RNI, conforme indicação da OMS, e a diferença maior entre uma técnica de coleta e outra foi de 0,6 , do paciente 80 , portador de prótese cardíaca, que ainda se mantém dentro do intervalo recomendado, conforme a Tabela 2.

A RNI foi calculada de acordo com a fórmula que se segue ${ }^{(16)}$, em que plasma controle (TP controle) foi igual a 12 segundos e o ISI da tromboplastina comercial foi de 1,4. O cálculo da RNI foi realizado no sentido da comparação, 
Valores recomendados pela Sociedade Internacional de Hemostasia e Trombose para 0 nivel de anticoagulação a ser

Tabela 2 atingido de acordo com a RNI ${ }^{(16)}$

\begin{tabular}{lcc}
\hline \multirow{2}{*}{ Situação clínica } & \multicolumn{2}{c}{ RNI } \\
& Alvo & Intervalo \\
\hline $\begin{array}{l}\text { Profilaxia de trombose venosa } \\
\text { e embolia pulmonar }\end{array}$ & 2,5 & 2 a 3 \\
Trombose venosa recorrente & 3 & 2 a 4 \\
Trombose arterial & 3,5 & 3 a 4,5 \\
Próteses valvares cardíacas & 3,5 & 3 a 4,5 \\
\hline
\end{tabular}

uma vez que esta é indicada para pacientes em uso de terapia anticoagulante oral ${ }^{(16)}$.

$$
\begin{gathered}
\mathrm{RNI}=\mathrm{R}^{|S|} \text {, } \\
\text { onde } \mathrm{R}=\frac{\text { TP paciente }}{\text { TP controle }}
\end{gathered}
$$

As médias obtidas (Tabela 3) dos valores encontrados na determinação do tempo de protrombina, tanto nas amostras coletadas com seringa quanto nas coletadas pelo sistema a vácuo, apresentaram variação mínima da ordem de 0,05. O valor crítico de $t_{0,05 ; G L=72}$ é de 1,99 para os pacientes hígidos e o resultado do teste $t$ calculado foi igual a 1,02, não-significativo $(p=0,311)$.

Para os pacientes em tratamento com anticoagulante oral o valor crítico de $t_{0,05 ; G L=16}$ é de 2,1199, e o resultado do teste $t$ calculado para o TP foi igual a 0,139 , não-significativo $(p=0,891)$, enquanto que o resultado do teste $t$ calculado para o RNI foi igual a -0,104, não-significativo ( $p$ = 0,981). Assim, os valores do TP e a RNI independem do método de coleta praticado. A distribuição das diferenças pareadas foi ligeiramente leptocúrtica, mas a aplicação de uma transformação logarítmica corrigiu esse problema e não afetou qualitativamente os resultados.

\section{Discussão}

O TP é um procedimento laboratorial que avalia os fatores II, V, VII e X da coagulação sangüínea, sendo que, destes, os fatores II, VII e X são vitamina K-dependentes ${ }^{(1,}$ 9). A determinação do TP é imprescindível na avaliação, no acompanhamento e na evolução de pacientes portadores de patologias variadas e também no monitoramento de pacientes que estão em uso de anticoagulante oral. Nesse caso, os resultados laboratoriais do TP e RNI expressos erroneamente levam a condutas clínicas inadequadas que podem levar o paciente a distúrbios hemorrágicos de graves dimensões ${ }^{(6,7)}$.

Os pacientes 6, 25, 37 e 55 estavam no início da terapia de profilaxia de trombose venosa, em fase progressiva para atingir o alvo, dentro do intervalo recomendado (Tabela 2), e nessa fase a precisão da RNI é menor. Nesse caso, o fator VII é aquele que apresenta diminuição imediata, contribuindo para o aumento do TP, pois o TP relaciona-se com os níveis plasmáticos de fatores vitamina $\mathrm{K}$ e não com os níveis plasmáticos da droga utilizada ${ }^{(16)}$.

Os dados obtidos dos 73 pacientes hígidos apresentam variações mínimas. Os resultados dos demais pacientes foram expressos também em RNI, pois todos faziam uso de anticoagulante oral. Mesmo assim, os resultados não se mostram discrepantes, não havendo variação entre os métodos empregados na obtenção da amostra na determinação de TP e RNI.

De acordo com os resultados obtidos e as análises estatísticas realizadas, demonstrou-se que não há diferença no resultado obtido das amostras provenientes das duas técnicas de coleta instituídas.

O emprego e a comparação das técnicas de coleta nesse trabalho permitiram e permitem oferecer ao paciente

\section{Tabela 3 Média, desvio padrão e teste $t$ pareado para comparação estatística de TP e RNI}

\begin{tabular}{lcccccc}
\hline & \multicolumn{2}{c}{ Pacientes hígidos } & \multicolumn{4}{c}{ Pacientes em uso de anticoagulante oral } \\
& TP vácuo & TP seringa & TP vácuo & TP seringa & RNI vácuo & RNI seringa \\
Média & 13,35 & 13,42 & 24,59 & 24,56 & 2,76 & 2,77 \\
Desvio padrão & 0,85 & 0,81 & 6,47 & 6 & 0,94 & 1,02 \\
Teste $t$ & $1,02(\mathrm{~ns})$ & & $0,139(\mathrm{~ns})$ & & $-0,104(\mathrm{~ns})$ & \\
\hline
\end{tabular}

ns $=$ não-significativo no nível de $5 \%$. 
mais um parâmetro de segurança na avaliação laboratorial do TP. Isso contribui para a uniformidade do resultado e seus cálculos, excluindo a forma de obtenção da amostra, especificamente nesse caso comparado, como um dos interferentes no resultado do analito.

Portanto, esses dois processos de obtenção de amostra para a avaliação do TP não interferem no resultado do analito, podendo-se usar com segurança qualquer uma das técnicas, ainda que o uso da técnica de coleta a vácuo tenha melhorado a qualidade dos resultados e minimizado os erros pré-analíticos.

\section{Agradecimentos}

Nossos agradecimentos aos Professores Dra. Fátima Mrue, Dr. José Alexandre Felizola Diniz Filho e Dr. Luis Murilo Martins de Araújo, pela colaboração e pelas orientações dispensadas a este trabalho

\section{References}

I. BARRANTES,A. B. El uso de reactivos estandarizados y el control de calidad en el método para el tiempo de protrombina. Rev Costar Cien Med, v. 3, p. 4I-50, 1982.

2. BULNES, P. Laboratorio en el tratamiento anticoagulante. Bol Hosp S J de Dios, v. 4I, n. 4, p. 263-8, 1994.

3. BESSELAAR, A. M. et al. Multicenter evaluation of a new capillary blood prothrombin time monitoring system. Blood Coagul Fibrin, v. 6, n. 8, p. 726-32, 1995.

4. BUSSEY, H. I. et al.A statistical and clinical evaluation of finger stick and routine laboratory prothrombin time measurements. Pharmacotherapy, v. 17, n. 5, p. 861-6, 1997.

5. CARRERAS, L. O.; KORDICH, L. C. Control del tratamiento con anticoagulantes orales. Sistema INR. Acta Bioq Clín Latinoa, v. XIX, n. 2, p. 179-86, 1985

6. FERREIRA, C. N. et al. Evaluation of the marker of hypercoagulability prothrombin fragment $\mathrm{F} \mathrm{I}+2$ in patients with mechanical or biological heart valve prostheses. Arq Bras Cardiol, v. 79 , n. 5, p. 505-9, 2002.

7. GANDUL, J.; HERNÁNDEZ, L; ARRAIGADA, L. Alteraciones de la coagulación en la hepatitis viral aguda. Rev Cubana de Med, v. 24, p. 1213-7, 1985.

8. GRINSPAN, S. Exámenes pré-operatorios para el estudio de la hemostasis. Rev Med Hondureña, v. 60, n. 3, p. 34-138, 1992.

9. GUITIÉRREZ, G. et al. Standardization Committee for Haematology. External Quality Assurance Program for General Hematology. Evaluation of the 1994 results. Sangre (Barc), v. 4I, n. 2, p.II5-23, 1996.

I0. HORSTI, J. Comparison of quick and owner prothrombin time with regard to the harmonization of the International Normalized Ratio (INR) system. Clin Chem Lab Med, v. 40, n. 4, p. 399-403, 2002.

I I. KAGAWA, K.; FUKUTAKE, K. Prothrombin time and its standardization: a potentiality to introduce INR method in criteria for disseminated intravascular coagulation. Rinsho Byori, v. 50, n. 3, p. 277-82, 2002.

12. KONDO H. et al. Hematology tests of blood anticoagulated with magnesium sulphate. Southeast Asian J Trop Med Public Health, v. 33, n. 2, p. 6-9, 2002.

13. LORENZI,T.F. Manual de Hematologia - Propedêutica e Clínica. 2 ed. Rio de Janeiro: Medsi, 1999. p. 64I.

14. LOURENÇO, D. M.; ALVES, E. D. C. Controle laboratorial da anticoagulação oral. Rev Assoc Méd Bras, v. 4I, n. 2, p. 103 $8,1995$.

15. MARZOUKA, E. Control del tratamiento con anticoagulantes orales y tiempo de protrombina. Rev Méd Chile, v. 199, n. 10, p. I |60-4, 1991.

I6. MIGUEL D. et al. Capillary whole blood testing by a new portable monitor. Comparison with standard determination of the international normalized ratio. Am J Clin Pathol, v. I20, n. I, p. 28-33, 2003.

17. MISCHKE, R.; DIEDRICH, M.; NOLTE, I. Sensitivity of different prothrombin time assays to factor VII deficiency in canine plasma. Vet J, v. I66, n. I, p. 79-85, 2003.

I 8. REIS, C.V. et al.Avaliação de coagulação, fibrinólise e proteína C em pacientes de risco e com doenças coronarianas.J Bras Patol Med Lab, v. 39, n. I, p. 7-13, 2003.

19. TERRA, P. Coagulação - Interpretação Clínica dos Testes Laboratoriais de Rotina. São Paulo: Atheneu, 2000.

20.VIEIRA, S. Introdução à Bioestatística. 3 ed. Rio de Janeiro: Editora Campus, 1998.
Endereço para correspondência Paulo Roberto de Melo Reis Universidade Católica de Coiás (LEPAH-LAS-CBB) - Área IV - Bloco H - Sala 209 Av. Universitária 1069 - Setor Universitário CEP 74605-010 - Coiânia-Co e-mail: melo.reis@ig.com.br ou pauloroberto@ucg.br 